\title{
ASYMPTOTIC REPRESENTATION : OF CERTAIN REAL INTEGRALS WITH PARAMETER-DEPENDENT LIMITS OF INTEGRATION
}

\author{
by E. W. ROSS, JR.
}

(Received 21st January 1961; Received in revised form 22nd May 1961)

\section{Introduction}

In this paper we consider a function $f(x)$ defined by

$$
f(x)=\int_{R(x)}^{b} G(t) e^{x N(t)} d t .
$$

All quantities are taken to be real, it is assumed that $R$ is a function of the variable $x, b$ is a constant, $N$ and $G$ are functions of the variable $t$ and all the functions are such that the integral (1) exists when $x$ is large enough. We wish to find an asymptotic representation of $f(x)$ as $x \rightarrow+\infty$, assuming that we are given certain information about the limiting behaviours of the functions $R, N$ and $G$.

Asymptotic representations of integrals like (1) but with fixed limits of integration are customarily found by Laplace's method. Erdélyi (1) gives a convenient discussion of this method, including further references, and proves the following general result about the case where $R(x)=a$, a constant with $a<b$.

Suppose that $N$ and $G$ are functions on $(a, b)$ for which the integral exists for all $x$ sufficiently large; $N$ is continuous at $a$, continuously differentiable with $d N / d t<0$ in $(a, a+\xi]$ and $N(t) \leqq N(a)-\Delta$ in $[a+\xi, b]$ where $\xi>0, \Delta>0$; $\int_{a+\xi}^{b}|G| d t$ exists; as $t \rightarrow a, d N / d t \sim-n(t-a)^{v-1}, G(t) \sim g(t-a)^{\gamma-1}$ where $\gamma, v$, $n>0$. Then as $x \rightarrow \infty$

$$
f(x) \sim(g / v) \Gamma(\gamma / v)[v /(n x)]^{\gamma / v} e^{x N(a)},
$$

where $\Gamma(T)$ denotes the Gamma-Function.

The condition on $\int_{a+\xi}^{b}|G| d t$ is not explicitly stated as a hypothesis in Erdélyi's theorem but is used in the proof, so we shall include it among the hypotheses.

In the present paper we shall apply Laplace's method to the more general case where $R$ depends on $x$. We shall assume that $R \rightarrow a+$ in a prescribed fashion as $x \rightarrow \infty$ and shall allow $G$ to have a more violent singularity at $a$ 
than is possible when $R=a$. The main theorem is stated in $\S 2$, some useful definitions and lemmas are established in $\S 3$, and the proof of the main theorem is given in $\S 4$. $\S 5$ contains a discussion of various special cases of the general result, and the resulting asymptotic formulas are summarised in \$.6.

The proof follows the general lines of the one given by Erdélyi in the case where the limits of integration are fixed.

\section{Statement of the Main Theorem}

At the outset we shall assume once and for all that $G(t), N(t)$ and $R(x)$ are such that the integral (1) exists for all $x$ sufficiently large and shall not in the theorem state conditions guaranteeing the existence of the integral.

Theorem: Suppose that

(i) as $x \rightarrow \infty$

where $r>0, \rho>0 \dagger$;

$$
R(x)-a \sim r x^{-\rho},
$$

(ii) $N$ is continuous at $a, N^{\prime}(t) \equiv d N / d t$ is continuous and $N^{\prime}(t)<0$ in $(a, a+\xi]$ for some $\xi>0$;

(iii) $N(t) \leqq N(a)-\Delta$ in $[a+\xi, b]$ for some $\Delta>0$;

(iv) $\int_{s}^{b}|G| d t$ exists for any s satisfying $a<s<b$;

(v) as $t \rightarrow a$,

$$
\begin{aligned}
N^{\prime}(t) & \sim-n(t-a)^{\nu-1} \\
G(t) & \sim g(t-a)^{\gamma-1},
\end{aligned}
$$

where $n>0, v>0$, but $g$ and $\gamma$ can be any real constants.

Then as $x \rightarrow \infty$,

$$
f(x) \sim(g / v)[v /(n x)]^{\gamma / v} e^{x N(a)} \Gamma[\gamma / v, \lambda(x)],
$$

where $\Gamma(\alpha, y)$ is the Incomplete Gamma-Function,

$$
\Gamma(\alpha, y)=\int_{y}^{\infty} u^{\alpha-1} e^{-u} d u
$$

and

$$
\lambda(x)=x\{N(a)-N[R(x)]\} .
$$

\section{Definitions and Lemmas}

In this section we shall record the definitions to be used in the subsequent sections and establish some very simple lemmas (under the conditions of the main theorem) that are needed in the proof of the main theorem.

$\dagger$ So far as the main theorem is concerned we need only assume that $R \rightarrow a+$ as $x \rightarrow \infty$. However, when we derive specific asymptotic formulas in $\S 5$, we shall limit ourselves to the situation described by (i), and so shall assume this from the outset. 


\section{Definitions:}

$$
\begin{aligned}
& F(x)=\int_{R(x)}^{a+\xi} G(t) e^{x[N(t)-N(a)]} d t \\
& u=N(a)-N(t) \\
& \psi(u)=-G(t) / N^{\prime}(t) \\
& \omega=N(a)-N(a+\xi)>0 \\
& \lambda(x)=x\{N(a)-N[R(x)]\} \\
& \alpha=\gamma / v \\
& \beta=(g / v)(v / n)^{\gamma / v} \\
& \Omega(x, \alpha)=x^{-\alpha} \Gamma[\alpha, \omega x]=x^{-\alpha} \int_{\omega x}^{\infty} s^{\alpha-1} e^{-s} d s \\
& \Lambda(x, \alpha)=x^{-\alpha} \Gamma[\alpha, \lambda(x)]=x^{-\alpha} \int_{\lambda(x)}^{\infty} s^{\alpha-1} e^{-s} d s . \\
& \phi(x, \alpha)=\beta \int_{\lambda / x}^{\omega} u^{\alpha-1} e^{-x u} d u \\
& E=n r^{v} / v \\
& \mu=1-\rho v \\
& \chi(x)=\Gamma[\alpha, \lambda(x)]
\end{aligned}
$$

Lemma 1: $\psi(u) \sim \beta u^{\alpha-1}$ as $u \rightarrow 0$.

Proof. Inserting (5) in (8) we have as $t \rightarrow a$

from which

$$
u=\int_{a}^{t}\left[-N^{\prime}(s)\right] d s \sim n \int_{a}^{t}(s-a)^{v-1} d s=(n / v)(t-a)^{v},
$$

$$
t-a \sim(u v / n)^{1 / v} \text {. }
$$

Combining (4) and (5) with (9) and then using (20), (12) and (13), we obtain finally

$$
\psi(u) \sim \beta u^{\alpha-1} .
$$

Lemma 2: $\phi(x, \alpha)=\beta[\Lambda(x, \alpha)-\Omega(x, \alpha)]$.

Proof. Setting $s=u x$ in (14) and (15), we obtain directly

$$
\beta[\Lambda-\Omega]=\beta\left[\int_{\lambda / x}^{\infty}-\int_{\infty}^{\infty}\right] u^{\alpha-1} e^{-x u} d u=\phi .
$$

Lemma 3: For any constant, $c>0, e^{c x} \Lambda(x, \alpha) \rightarrow \infty$ as $x \rightarrow \infty$. 
Proof. We put $s=u x$ in (15) and find

$$
e^{c x} \Lambda(x, \alpha)=\int_{\lambda / x}^{\infty} u^{\alpha-1} e^{(c-u) x} d u
$$

From (11) and (3) $\lambda / x \rightarrow 0$ as $x \rightarrow \infty$. Hence we can choose $x$ so large that $\lambda / x<c-\delta$, where $\delta$ satisfies $0<\delta<c$. Then

$$
e^{c x} \Lambda>\int_{\lambda / x}^{c-\delta} u^{\alpha-1} e^{(c-u) x} d u
$$

and, since $c-u>\delta$ in this integral,

$$
e^{c x} \Lambda>e^{\delta x} \int_{\lambda / x}^{c-\delta} u^{\alpha-1} d u
$$

The last integral is positive and bounded away from zero for $x$ large enough, hence $e^{c x} \Lambda \rightarrow \infty$.

Lemma 4: (a) $\Omega / \Lambda \rightarrow 0$ as $x \rightarrow \infty$.

(b) $e^{c x}(\Lambda-\Omega) \rightarrow \infty$ as $x \rightarrow \infty$ for any $c$ with $0<c<\omega$.

Proof. From the asymptotic representation for the Incomplete Gamma Function (see (2)) we have as $x \rightarrow \infty$

$$
\Omega=e^{-\operatorname{cox}} O\left(x^{-1}\right)
$$

and so

$$
\Omega / \Lambda=O\left(x^{-1}\right) / e^{\omega x} \Lambda \rightarrow 0
$$

by Lemma 3 since $\omega>0$. This proves part (a). To prove (b), we have from (21) as $x \rightarrow \infty$

$$
e^{c x} \Omega=e^{(c-\omega) x} O\left(x^{-1}\right) \rightarrow 0
$$

and the proof then follows from the fact that $e^{c x} \Lambda \rightarrow \infty$ by Lemma 3 .

Lemma 5: If $c$ satisfies $0<c<\omega, \int_{c}^{\omega}|\psi(u)| d u$ exists.

Proof. Because of (ii) one and only one $c^{*}$ exists satisfying $c=N(a)-N\left(c^{*}\right)$ and $a<c<a+\xi$. From (8) and (9) then,

$$
\int_{u=c}^{\infty}|\psi(u)| d u=\int_{t=c^{*}}^{a+\xi}|G(t)| d t=\int_{t=c^{*}}^{b}|G| d t-\int_{a+\xi}^{b}|G| d t
$$

and the last two integrals exist according to (iv).

\section{Proof of the Main Theorem}

We observe first that for $x$ sufficiently large $a<R<a+\xi$. The integral is split as follows:

$$
f(x)=\int_{R}^{a+\xi} G e^{x N} d t+\int_{a+\xi}^{b} G e^{x N} d t
$$


ASYMPTOTIC REPRESENTATION OF REAL INTEGRALS 181

The second of these two integrals may be estimated immediately from condition (iii).

$$
\left|\int_{a+\xi}^{b} G e^{x N} d t\right| \leqq \int_{a+\xi}^{b}|G| e^{x[N(a)-\Delta]} d t=e^{x N(a)} e^{-x \Delta} \int_{a+\xi}^{b}|G| d t .
$$

Condition (iv) states that $\int_{a+\xi}^{b}|G| d t$ exists, hence

$$
\left|\int_{a+\xi}^{b} G e^{x N} d t\right|=e^{x N(a)} O\left(e^{-x \Delta}\right)
$$

Let us now turn our attention to the first integral in (22). From (7)

$$
\int_{R}^{a+\xi} G e^{x N} d t=e^{x N(a)} F(x) .
$$

If we introduce in $F(x)$ the transformation defined by (8)-(11), the result is

$$
F(x)=\int_{u=\lambda / x}^{\infty} \psi(u) e^{-x u} d u .
$$

The next step in the proof will be to show that $F \dot{\sim} \beta \Lambda$ as defined by (13) and (15).

From (25) and (16)

$$
\begin{aligned}
|F-\phi| & \leqq \int_{\lambda / x}^{\infty}\left|\psi(u)-\beta u^{\alpha-1}\right| e^{-x u} d u \\
& \leqq\left[\int_{\lambda / x}^{c}+\int_{c}^{\infty}\right]\left|\psi(u)-\beta u^{\alpha-1}\right| e^{-x u} d u,
\end{aligned}
$$

where $c$ is a number satisfying $0<c<\omega$ and such that in $0<u<c$

$$
\left|\psi(u)-\beta u^{\alpha-1}\right|<\varepsilon|\beta| u^{a-1}
$$

for any $\varepsilon>0$. That such a number, $c$, exists follows from the fact that $\psi \sim \beta u^{\alpha-1}$ as $u \rightarrow 0$ according to Lemma 1 . Since $\lambda / x \rightarrow 0$ as $x \rightarrow \infty$, (27) holds true in $\lambda / x<u<c$ for $x$ sufficiently large, and so from (27) and (16)

$$
\int_{\lambda / x}^{c}\left|\psi(u)-\beta u^{\alpha-1}\right| e^{-x u} d u<\varepsilon|\beta| \int_{\lambda / x}^{c} u^{\alpha-1} e^{-x u} d u<\varepsilon|\phi| .
$$

In the second integral of (26) we have

$$
\int_{c}^{\infty}\left|\psi(u)-\beta u^{\alpha-1}\right| e^{-x u} d u \leqq e^{-c x}\left[\int_{c}^{\infty}|\psi(u)| d u+|\beta| \int_{c}^{\infty} u^{\alpha-1} d u\right] \text {. }
$$

The first integral on the right exists according to Lemma 5 , and the second obviously does also. Thus an upper bound (say $M_{1}$ ) exists for the bracketed expression in (29). If we use (28) and (29) in (26) and take account of Lemma 2, we obtain

$$
|F-\phi| /|\phi|<\varepsilon+\frac{M_{1}}{|\beta| e^{c x}(\Lambda-\Omega)}
$$


Then by Lemma $4(b)$, the second term on the right approaches zero and so $F \sim \phi$ as $x \rightarrow \infty$. By Lemmas 2 and $4(a)$

$$
\phi=\beta \Lambda[1-(\Omega / \Lambda)] \sim \beta \Lambda,
$$

and so we get that $F \sim \beta \Lambda$, or

$$
F=\beta \Lambda\left(1+F_{1}\right)
$$

where $F_{1} \rightarrow 0$ as $x \rightarrow \infty$.

The proof of the theorem is completed by putting (23), (24) and (30) into (22) to obtain

$$
\begin{aligned}
f(x) e^{-x N(a)} & =\beta \Lambda\left(1+F_{1}\right)+O\left(e^{-x \Delta}\right) \\
& =\beta \Lambda\left[1+F_{1}+\frac{O\left(e^{-x \Delta}\right)}{\beta \Lambda}\right] .
\end{aligned}
$$

The last term in the bracket approaches zero by Lemma 3, and so

$$
f(x) \sim \beta \Lambda(x, \alpha) e^{x N(a)} .
$$

When account is taken of definitions (11), (12), (13) and (15), the conclusion (6) of the main theorem is reached.

\section{Discussion}

In order to obtain specific asymptotic formulas for $f(x)$ in various cases, it is necessary first to find an asymptotic representation of $\lambda(x)$. To do this we observe that from (11) and (4)

$$
\lambda(x) / x=-\int_{a}^{R(x)} N^{\prime}(t) d t \sim(n / v)[R(x)-a]^{v},
$$

and so because of (3), (17) and (18)

$$
\lambda(x) \sim\left(n r^{v} / v\right) x^{1-\rho v}=E x^{\mu}
$$

as $x \rightarrow \infty$. We shall now use the known properties of the Incomplete Gamma Function to find asymptotic formulas for $f(x)$. In doing this a number of different cases may be distinguished, depending on the values of the parameters $\rho v$ and $\alpha=\gamma / v$. The cases in which $\lambda(x)$ approaches a finite value as $x \rightarrow \infty$, i.e. when $\rho v \geqq 1$, are straightforward. More care is necessary when $\lambda \rightarrow \infty$, or $\rho v<1$, as in this case the function obtained by substituting the asymptotic representation of $\lambda(x)$ as $x \rightarrow \infty$ into that of $\Gamma(\alpha, \lambda)$ as $\lambda \rightarrow \infty$ is not necessarily the asymptotic representation of the function $\chi(x)=\Gamma[\alpha, \lambda(x)]$.

We consider first the cases where $\rho v \geqq 1$. In particular if $\rho v=1, \lambda \rightarrow(n / v) r^{v}>0$ as $x \rightarrow \infty$, and so we obtain in this simple situation just

$$
f(x) \sim(g / v) \Gamma\left(\gamma / v, n r^{v} / v\right)[v /(n x)]^{\gamma / v} e^{x N(a)} .
$$


If $\rho v>1, \lambda \rightarrow 0$ as $x \rightarrow \infty$, and the asymptotic behaviour of $\Gamma(\alpha, \lambda)$ depends on $\alpha$ and is given in (2) as

$$
\begin{aligned}
\Gamma(\alpha, \lambda) & =\Gamma(\alpha)+O\left(\lambda^{\alpha}\right) & & \text { if } \quad \alpha>0 \\
& =-\ln \lambda+O(1) & & \text { if } \quad \alpha=0 \\
& =\frac{\lambda^{\alpha}}{(-\alpha)}[1+O(\lambda)]+O(1) & & \text { if } \quad \alpha<0,
\end{aligned}
$$

where. $\Gamma(\alpha)=\Gamma(\alpha, 0)$ is the (Complete) Gamma Function. When we insert the asymptotic dependence of $\lambda$ upon $x$ from (31), we obtain

$$
\begin{aligned}
\Gamma(\gamma / v, \lambda) & \sim \Gamma(\gamma / v) & & \text { if } \gamma / v>0 \\
& \sim(\rho v-1) \ln x & & \text { if } \gamma / v=0 \\
& \sim \frac{r^{\gamma}(n / v)^{\gamma / v}}{(-\gamma / v)} x^{\gamma(v-1-\rho)} & & \text { if } \gamma / v<0 .
\end{aligned}
$$

The asymptotic representations of $f(x)$ are obtained by substituting these asymptotic representations of $\Gamma(\gamma / v, \lambda)$ into (6). We obtain finally for $\rho v>1$

$$
\begin{aligned}
f(x) & \sim(g / v) \Gamma(\gamma / v)[v /(n x)]^{\gamma / v} e^{x N(a)} & & \text { if } \gamma / v>0 \\
& \sim(g / v)(\rho v-1)(\ln x) e^{x N(a)} & & \text { if } \gamma / v=0 \\
& \sim g r^{\gamma}(-\gamma)^{-1} x^{-\gamma \rho} e^{x N(a)} & & \text { if } \gamma / v<0 .
\end{aligned}
$$

By considering the remainders in the original asymptotic representations we may verify that it is permissible to substitute asymptotic representations as we have done in all the foregoing cases.

The first of the above formulas is just the result (2) given by Erdélyi (1) for the case where $R=a$ and $\gamma>0$. Since the parameter $\rho$ characterises the rapidity with which the lower limit of integration approaches $a$ as $x \rightarrow \infty$, we conclude that the asymptotic representation is too insensitive to distinguish between the situations where $R=a$ and $R-a=O\left(x^{-\rho}\right)$ and $\rho>1 / v$.

We consider now the case $0<\rho v<1$, for which $\lambda \rightarrow \infty$ as $x \rightarrow \infty$. To see that in this case it is not permissible merely to substitute the asymptotic representation of $\lambda(x)$ into that of $\Gamma(\alpha, \lambda)$, we combine the asymptotic representation of $\Gamma(\alpha, \lambda)$ as $\lambda \rightarrow \infty$ (see (2)),

$$
\Gamma(\alpha, \lambda)=\lambda^{\alpha-1} e^{-\lambda}\left[1+0\left(\lambda^{-1}\right)\right],
$$

with that of $\lambda(x)$ given by (31),

$$
\lambda(x)=E x^{\mu}\left[1+\lambda_{1}(x)\right]
$$

where $\lambda_{1}(x) \rightarrow 0$ as $x \rightarrow \infty$ and $\mu>0$ in the present case. This leads to $\chi(x) \equiv \Gamma[\alpha, \lambda(x)]=\left\{\left(E x^{\mu}\right)^{\alpha-1} e^{-E x^{\mu}}\right\} \times\left[1+O\left(\lambda_{1}\right)\right]\left[1+O\left(x^{-\mu}\right)\right] e^{-E x^{\mu} \lambda_{1}(x)}$.

If the result

$$
\chi(x) \sim\left(E x^{\mu}\right)^{\alpha-1} e^{-E x^{\mu}}
$$


is to be true, than we must have $x^{\mu} \lambda_{1}(x) \rightarrow 0$ as $x \rightarrow \infty$, and this does not necessarily follow from $\lambda_{1} \rightarrow 0$.

It is clear from (32) that we must retain more terms in the asymptotic representation of $\lambda(x)$ if we want to find a correct representation of $\chi(x)$ when $\mu>0$. We have assumed in the main theorem that

$$
\begin{aligned}
R-a & =r x^{-\rho}\left[1+R_{1}(x)\right] \\
N^{\prime}(t) & =-n(t-a)^{p-1}\left[1+N_{1}(t)\right],
\end{aligned}
$$

where $R_{1}(x) \rightarrow 0$ as $x \rightarrow \infty$ and $N_{1}(t) \rightarrow 0$ as $t \rightarrow a$; we shall now assume in addition that the remainders, $R_{1}$ and $N_{1}$, possess asymptotic expansions in powers of $x^{-\sigma}$ and $(t-a)^{\delta}$ (where $\sigma>0, \delta>0$ ) to any desired number of terms, say $c_{1}$ and $c_{2}$ respectively, $\dagger$ i.e.

$$
\begin{aligned}
& R_{1}=\sum_{q=1}^{c_{1}} T_{q} x^{-\sigma q}+O\left(x^{-\sigma c_{1}}\right) \\
& N_{1}=\sum_{p=1}^{c_{2}} U_{p}(t-a)^{p \delta}+O\left[(t-a)^{\delta c_{2}}\right] .
\end{aligned}
$$

Using these assumptions in the formula

$$
\lambda(x)=x \int_{t=a}^{R}\left[-N^{\prime}(t)\right] d t
$$

and carrying out the integration, we obtain

where

$$
\lambda(x)=E x^{\mu}\left[1+R_{1}(x)\right]^{v}\left\{\sum_{p=0}^{c_{2}} V_{p} x^{-p \rho \delta}\left[1+R_{1}(x)\right]^{p \delta}+o\left(x^{-\rho c_{2} \delta}\right)\right\},
$$

$$
\left.\begin{array}{l}
V_{o}=1 \\
V_{p}=U_{p} v r^{p \delta} /(v+p \delta), \quad p \geqq 1
\end{array}\right\} .
$$

Using the binomial expansion, we find after some manipulation

where

$$
\begin{gathered}
{\left[1+R_{1}(x)\right]^{v}=\sum_{m=0}^{c_{1}} A_{m} x^{-\sigma m}+o\left(x^{-\sigma c_{1}}\right)} \\
{\left[1+R_{1}(x)\right]^{p \delta}=\sum_{s=0}^{c_{1}} B_{s}(p) x^{-\sigma s}+o\left(x^{-\sigma c_{1}}\right),}
\end{gathered}
$$

$$
\begin{aligned}
A_{0} & =1 \\
A_{m} & =\sum_{h=1}^{m}\left(\begin{array}{l}
v \\
h
\end{array}\right) \sum_{m}\left(\prod_{j=1}^{h} T_{i_{j}}\right) \quad \text { for } m \geqq 1 \\
B_{0} & =1 \\
B_{s}(p) & =\sum_{h=1}^{s}\left(\begin{array}{c}
p \delta \\
h
\end{array}\right) \sum_{s}\left(\prod_{j=1}^{h} T_{i j}\right) \quad \text { for } s \geqq 1 .
\end{aligned}
$$

$\dagger c_{1}$ and $c_{2}$, do not need to be assumed arbitrarily large; see the remarks preceding (43). 
ASYMPTOTIC REPRESENTATION OF REAL INTEGRALS 185

In these formulas $\Sigma_{m}$ denotes that the summation is over all terms with $\sum_{j=1}^{h} i_{j}=m$ and $i_{j}$ taking integer values satisfying $1 \leqq i_{j} \leqq c_{1} . \quad \Sigma_{s}$ denotes a similar summation except that $\sum_{j=1}^{h} i_{j}=s$. If these results are used in (35). we obtain

where

$$
\begin{aligned}
\lambda(x) & =E x^{\mu}\left[1+\sum_{p, m, s} W_{p m s} x^{-\sigma(m+s)-p p \delta}+o\left(x^{-z}\right)\right] \\
& =E x^{\mu}\left[1+\lambda_{1}\right],
\end{aligned}
$$

$$
W_{p m s}=V_{p} A_{m} B_{s}(p)
$$

the summation is over all integer values of $p, m$ and $s$ satisfying

$$
\begin{aligned}
& p+m+s \geqq 1 \\
& 0 \leqq m, s \leqq c_{1}, \quad 0 \leqq p \leqq c_{2}
\end{aligned}
$$

and $\tau$ is the smaller of $\rho c_{2} \delta$ and $c_{1} \sigma$. If we insert the above asymptotic expansion for $\lambda(x)$ into (32), we find

$$
\begin{aligned}
\chi(x)=\left(E x^{\mu}\right)^{\alpha-1} & {\left[1+O\left(\lambda_{1}\right)\right]\left[1+O\left(x^{-\mu}\right)\right] } \\
& \times \exp \left\{-E\left[x^{\mu}+\sum_{p, m, s} W_{p m s} x^{\mu-\sigma(m+s)-p p s}+o\left(x^{\mu-\tau}\right)\right]\right\} .
\end{aligned}
$$

The summation in this formula can be split so that

$$
\sum_{p, m, s}=\Sigma_{a}+\Sigma_{r}
$$

where $\Sigma_{a}$ is extended over all integer indices satisfying

$$
\begin{aligned}
& \mu-\sigma(m+s)-p \rho \delta \geqq 0 \\
& p+m+s \geqq 1 \\
& 0 \leqq p \leqq c_{2}, \quad 0 \leqq m, s \leqq c_{1},
\end{aligned}
$$

and $\Sigma_{r}$ is extended over all integer indices satisfying

$$
\begin{aligned}
& \mu-\sigma(m+s)-p \rho \delta<0 \\
& p+m+s \leqq 1 \\
& 0 \leqq p \leqq c_{2}, \quad 0 \leqq m, s \leqq c_{1} .
\end{aligned}
$$

As $x \rightarrow \infty$, it is clear from (38) and (41) that $\Sigma_{r} \rightarrow 0$ and also that the remainder term $o\left(x^{\mu-\eta}\right)$ approaches zero if $c_{1}$ and $c_{2}$ are large enough. Then we obtain as $x \rightarrow \infty$.

$$
\chi(x) \sim\left(E x^{\mu}\right)^{a-1} \exp \left[-E\left(x^{\mu}+\Sigma_{a}\right)\right]
$$

It is now possible to see that we do not need to assume that $c_{1}$ and $c_{2}$ are 
arbitrarily large but only that they are large enough so that $\Sigma_{a}$ contains all the terms of $\sum_{p, m, s}$ not approaching zero as $x \rightarrow \infty$. The following inequalities are sufficient to ensure this:

$$
\left.\begin{array}{l}
c_{2} \geqq \mu / \rho \delta=(1-\rho v) / \rho \delta \\
c_{1} \geqq \mu / \sigma=(1-\rho v) / \sigma
\end{array}\right\} .
$$

It is also worth observing that if $\Sigma_{a}$ is vacuous, then (34), which is obtained by substituting (31) into (32), is in fact correct. Sufficient conditions for this to be so are found by setting $p=1, m=s=0$ and $p=s=0, m=1$ in (41). Hence (34) is true if the two inequalities

are met.

$$
\begin{aligned}
\rho \delta>\mu & =1-\rho v \\
\sigma>\mu & =1-\rho v
\end{aligned}
$$

Combining (42) with (6), and using the definitions of $E$ and $\mu$, we obtain for the case $0<\rho v<1$

$$
f(x) \sim g n^{-1} r^{\gamma-v} x^{-1+\rho(v-\gamma)} \exp \left[x N(a)-n r^{v} v^{-1}\left(x^{1-\rho v}+\Sigma_{a}\right)\right] .
$$

For convenience we include here a list of the first five of the coefficients $A_{m}$; the coefficient $B_{s}$ is identical with $A_{s}$ except that the binomial coefficient $\left(\begin{array}{l}v \\ h\end{array}\right)$ must be replaced by $\left(\begin{array}{c}p \delta \\ h\end{array}\right)$. With these coefficients and the formulas (36) for $V_{p}$, enough of the coefficients $W_{p m s}$ may be calculated by (37) to cover most cases of probable interest. The coefficients $A_{0}, \ldots A_{4}$ are

$$
\begin{aligned}
& A_{0}=1 \\
& A_{1}=\left(\begin{array}{l}
v \\
1
\end{array}\right) T_{1} \\
& A_{2}=\left(\begin{array}{l}
v \\
1
\end{array}\right) T_{2}+\left(\begin{array}{l}
v \\
2
\end{array}\right) T_{1}^{2} \\
& A_{3}=\left(\begin{array}{l}
v \\
1
\end{array}\right) T_{3}+\left(\begin{array}{l}
v \\
2
\end{array}\right)\left(2 T_{1} T_{2}\right)+\left(\begin{array}{l}
v \\
3
\end{array}\right) T_{1}^{3} \\
& A_{4}=\left(\begin{array}{l}
v \\
1
\end{array}\right) T_{4}+\left(\begin{array}{l}
v \\
2
\end{array}\right)\left(2 T_{1} T_{3}+T_{2}^{2}\right)+\left(\begin{array}{l}
v \\
3
\end{array}\right)\left(3 T_{1}^{2} T_{2}\right)+\left(\begin{array}{l}
v \\
4
\end{array}\right) T_{1}^{4} .
\end{aligned}
$$

\section{Summary of Results}

We consider $f(x)$ given by

$$
f(x)=\int_{R(x)}^{b} G(t) e^{x N(t)} d t
$$


ASYMPTOTIC REPRESENTATION OF REAL INTEGRALS 187

Under the conditions of the main theorem, if

$$
\begin{array}{ll}
R(x)-a \sim r x^{-\rho} & \text { as } x \rightarrow \infty ; r>0, \rho>0, \\
N^{\prime}(t) \sim-n(t-a)^{v-1} & \text { as } t \rightarrow a ; n>0, v>0, \\
G(t) \sim g(t-a)^{\gamma-1} & \text { as } t \rightarrow a,
\end{array}
$$

( $g$ and $\gamma$ may be any real numbers), then as $x \rightarrow \infty$, the asymptotic behaviour of $f(x)$ is as follows:

For $\rho v>1, \gamma>0, \quad f(x) \sim(g / v) \Gamma(\gamma / v)[v /(n x)]^{\gamma / v} e^{x N(a)}$

$$
\begin{array}{ll}
\gamma=0, & f(x) \sim(g / v)(\rho v-1)(\ln x) e^{x N(a)} \\
\gamma<0, & f(x) \sim g r^{\gamma}(-\gamma)^{-1} x^{-v \rho} e^{x N(a)}
\end{array}
$$

For $\rho v=1 \quad f(x) \sim(g / v) \Gamma\left(\gamma / v, n r^{v} / v\right)[v /(n x)]^{y / v} e^{x N(a)}$

For $\rho v<1 \quad f(x) \sim g n^{-1} r^{y-v} x^{-1+\rho(v-\gamma)} \exp \left\{x N(a)-n r^{v} v^{-1}\left(x^{1-\rho v}+\Sigma_{a}\right)\right\}$, where $\Sigma_{a}$ is defined by (39) and (40).

\section{Acknowledgment}

This work originally appeared as a Watertown Arsenal Laboratories Report, and the author expresses his thanks to the U.S. Army Office of Ordnance Research for permission to publish it.

\section{REFERENCES}

(1) A. ErdélyI, Asymptotic Expansions (New York, Dover Publication, 1956), p. $37 \mathrm{ff}$.

(2) Bateman Manuscript Project, Higher Transcendental Functions, Volume II (New York, McGraw-Hill, 1953), pp. 133-145.

WATERTOWN ARSENAL

WATERTOWN, MasS., U.S.A.

and

King's COLleGE

NEWCASTLE-UPON-TYNE 